\title{
Adverse childhood events and psychosis in bipolar affective disorder
}

Rachel Upthegrove, Christine Chard, Lisa Jones, Katherine Gordon-Smith, Liz Forty, lan Jones and Nick Craddock

\section{Background}

There has been increasing interest in the association between childhood trauma and psychosis. Proposals for potential mechanisms involved include affective dysregulation and cognitive appraisals of threat.
Aims
To establish if, within bipolar disorder, childhood events show a significant association with psychosis, and in particular with symptoms driven by dysregulation of mood or with a persecutory content.

\section{Method}
Data on lifetime-ever presence of psychotic symptoms were determined by detailed structured interview with case-note review $(n=2019)$. Childhood events were recorded using a self-report questionnaire and case-note information.

\section{Results}

There was no relationship between childhood events, or childhood abuse, and psychosis per se. Childhood events were not associated with an increased risk of persecutory or other delusions. Significant associations were found between childhood abuse and auditory hallucinations, strongest between sexual abuse and mood congruent or abusive voices. These relationships remain significant even after controlling for lifetime-ever cannabis misuse.

\section{Conclusions}

Within affective disorder, the relationship between childhood events and psychosis appears to be relatively symptomspecific. It is possible that the pathways leading to psychotic symptoms differ, with delusions and non-hallucinatory symptoms being influenced less by childhood or early environmental experience.

\section{Declaration of interest} None.
In recent years a number of studies have investigated an association between the presence of psychotic symptoms and a history of childhood trauma. A recent meta-analysis of 36 studies concluded childhood adversity increases the risk of psychosis nearly threefold. ${ }^{1}$ Some authors now propose a causal relationship between these early childhood events and the subsequent development of schizophrenia, ${ }^{2,3}$ and this association has influenced key cognitive models of psychosis. One example is the proposal that adverse experiences in childhood will lead to the development of negative schemas of the self and the world (the self as vulnerable and others as dangerous) that facilitate the development of paranoid delusions. ${ }^{4}$ Furthermore, Birchwood et al suggest that childhood experience of social adversity leads to the development of negative schemas involving social humiliation and subordination, which in turn may fuel paranoia. ${ }^{5}$ Alternatively, it is proposed within biological models of schizophrenia that the experience of abuse creates vulnerability to psychosis through heightened stress reactivity and cortisol dysfunction., ${ }^{6,7}$ In addition, affective dysfunction following childhood trauma is increasingly highlighted as a mechanism through which psychosis develops. ${ }^{3,8}$

However, this analysis, and much of the literature to date, focuses on non-affective psychosis or data from population-based studies with subclinical psychotic-like experiences. ${ }^{1,2,9-11}$ We know that childhood trauma is also associated with a wide number of adverse outcomes, for example depression, suicidal behaviour, personality disorder and bipolar disorder. ${ }^{12}$ Affective dysfunction is also proposed as a mechanism to explain these associations. For example, Etain et al suggest that a dual role of genetic and environmental influences of socially and morally inappropriate rewards and parental attitudes during childhood induces affective dysregulation in the developing child that precedes the development of bipolar disorder. ${ }^{13,14}$ Thus, given that childhood trauma is proposed as a risk factor for psychosis and affective dysfunction, it is surprising that few studies have investigated the role of childhood trauma in psychotic symptoms as part of an affective disorder to date. In addition, childhood trauma itself encompasses many experiences, but few studies have investigated specific life events in detail. Bentall et al looked at this issue in a large population-based sample and found that sexual abuse was associated only with hallucinations, whereas being brought up in institutional care was associated with 'paranoia.. They proposed the specific associations observed were consistent with current psychological theories about the origins of hallucinations and paranoia. ${ }^{15}$ Our study sought to build and expand the current evidence base by exploring the association between a range of adverse childhood events and the presence of psychotic symptoms in a very large, well-characterised sample of patients with bipolar disorder. We hypothesised that for individuals with affective disorder, childhood trauma would show a significant association with psychosis, and in particular with psychotic symptoms coupled with dysregulation of mood (i.e. mood congruent delusions and hallucinations) and with persecutory or abusive content.

\section{Method}

The study was part of our ongoing programme of research into the genetic and non-genetic determinants of bipolar disorder and related mood disorders (Bipolar Disorder Research Network, BDRN; www.bdrn.org) which has UK National Health Service (NHS) Research Ethics Committee approval and local research and development approval in all participating NHS trusts/health boards. In light of the potential distress caused by the subject matter involved in this study, ethical approval and study methods included information on how to access support helplines from the 
charity Bipolar UK (www.bipolaruk.org.uk/), a user-led charity, and telephone access to the study team that included experienced clinicians with the ability to access local help as needed. The Childhood Life Events Questionnaire (CLEQ, see online supplement DS1) was not asked until towards the end of the $1.5 \mathrm{~h}$ interview to ensure researchers had time to establish rapport with participants before asking about events occurring in their childhood. Researchers also took the time to explain to participants why these events are being asked about. In many cases, events occurring in childhood were disclosed by the participant to the researcher before the CLEQ was asked as participants felt these events had played a role in the course of their mood illness. Throughout the interview, participants could ask the interviewer to move onto another question, take a break or end the interview at any time. Each researcher had sufficient training and experience to provide the necessary support and advice to participants who experienced distress following the interview.

\section{Participants}

Participants were recruited via both systematic and non-systematic recruitment methods. Participants were recruited systematically through NHS psychiatric services (community mental health teams and lithium clinics) and non-systematically using advertisements for volunteers via the research team websites, leaflets, posters, media coverage and Bipolar UK. The research programme inclusion criteria required participants to be: (a) 18 years or over, (b) able to provide written informed consent, (c) their affective disorder not to be the result of alcohol/substance misuse or dependence, medical illness or medication, (d) not biologically related to another study participant, and (e) UK White British (because of the research programme focus on genetic analysis). This current study was conducted on a subset of participants who had a DSM-IV ${ }^{16}$ diagnosis of type I bipolar disorder for whom data on adverse childhood events had been collected. Given the focus on affective disorder and psychosis, we excluded those with a diagnosis of type II bipolar disorder, where psychotic symptoms would be less likely to occur. ${ }^{17}$

\section{Data collection}

Clinical data were collected using the Schedules for Clinical Assessment in Neuropsychiatry (SCAN) semi-structured interview. ${ }^{18}$ This was supplemented by reviewing participants' psychiatric case notes. Data were combined to make best-estimate lifetime-ever diagnoses according to DSM-IV and to rate lifetime-ever clinical characteristics. This included age at illness onset, lifetime alcohol and substance use and the lifetime presence/absence of specific delusions (including persecutory, grandiose, depressive, nihilistic, guilt and reference); auditory hallucinations (including mood congruent hallucinations, accusatory/abusive and running commentary) and visual hallucinations (including mood congruent visual hallucinations). Interrater reliability was high. Mean kappa statistics were 0.85 for DSM-IV diagnoses and ranged between 0.81 and 0.99 for other key clinical categorical variables; mean intraclass correlation coefficients were between 0.91 and 0.97 for key clinical continuous variables.

Adverse childhood events were assessed by asking participants, via the CLEQ, if they had experienced one or more of a list of 13 adverse childhood events before the age of 16, including separation/remarriage of parents, the death of a parent, friend or sibling, admission to hospital/jail sentence of a parent and being suspended from school. Information specifically for childhood abuse events were collected from a number of sources, including the CLEQ. In the CLEQ, childhood abuse and bullying are not specifically asked about, however participants are given the opportunity to disclose additional events by being asked 'Are there any other significant life events you experienced as a child that are not mentioned above?'. Case notes were also reviewed for any mention of adverse child events including abuse and bullying. In addition, participants completed the Brief Life Events Questionnaire (BLEQ), with an open question included asking for details of any other life event - this was also examined for evidence of childhood events. ${ }^{19}$

\section{Statistical analysis}

Individuals were grouped according the lifetime presence or absence of psychosis as defined by the presence or absence of any type of delusion or hallucination. Demographic and clinical characteristics of the groups with and without psychosis were compared using a chi-squared test of association and independent samples $t$-test as appropriate. We examined the association of childhood events with psychosis in general and then specific psychotic symptoms, the presence or absence of grandiose delusions, depressive delusions (including nihilistic delusions, delusions of guilt and/or delusions of poverty) and persecutory delusions, mood congruent auditory and visual hallucinations, and second-person abusive/accusatory or third-person/running commentary auditory hallucinations.

Individuals were grouped as either having experienced any adverse childhood event or not. The 13 types of childhood events were then grouped into the five most frequent categories: childhood abuse, death of a loved one, victimising events (including bullying), family disruption (including parental separation) and difficulties at school. Childhood abuse was then further classified into sexual, physical and emotional abuse. Chi-square tests of association were used to explore the relationship between lifetime history of experiencing childhood events, psychosis and specific psychotic symptoms. For categories with significant association, odds ratios (ORs) with 95\% confidence intervals were calculated, using forward stepwise logistic regression. Odds ratios were adjusted to account for lifetime-ever cannabis use (as there is considerable evidence of the role of this confounding variable in the mediation of trauma and psychosis), ${ }^{20,21}$ gender, age at interview and age at onset. Previous studies have commented on the increased risk of childhood sexual abuse and psychosis in females. ${ }^{22}$ Data analysis was performed using the SPSS 20.0 package for Windows.

\section{Results}

\section{Demographic and clinical characteristics}

The final sample consisted of 2019 participants with a mean age of 47 years; $70 \%(n=1415)$ were female and 70\% $(n=1408)$ had a lifetime history of any psychosis (Table 1$)$. The participants with psychosis were more likely to be female $\left(\chi^{2}(1)=6.5, P=0.01\right)$, younger at interview $(t=-4.7, P=0.007)$ and have an earlier age at illness onset $(t=-3.08, P=0.002)$. A total of $74 \%$ $(n=1499)$ of participants reported experiencing one or more adverse childhood event. In addition, 17\% $(n=349)$ reported two different categories of adverse event, 9\% $(n=177)$ reported three and $3 \%(n=66)$ reported four or more. A history of lifetime-ever cannabis use was significantly associated with any childhood event $\left(\chi^{2}=10.6, P=0.001\right)$.

\section{Childhood events and association with psychotic symptoms}

Initial analysis showed that having experienced any childhood event, or childhood abuse, was not significantly associated with 


\begin{tabular}{|c|c|c|c|c|}
\hline & Total sample $(n, 2019)$ & Psychosis lifetime ever & No psychosis & Significance \\
\hline Age (mean, s.d.) & $47(12.13)$ & $46(11.81)$ & $48(12.63)$ & $t=-4.7, P=0.007^{1}$ \\
\hline Female $(n, \%)$ & $1415(70)$ & $1011(50)$ & $404(20)$ & $\chi^{2}($ d.f. $)=6.5(1), P=0.01$ \\
\hline Age of onset $^{2}$ (mean, s.d.) & $23(9.4)$ & $23(8.8)$ & $24(10.66)$ & $t=-3.08, P=0.002^{1}$ \\
\hline History of admissions ( $n, \%)$ & $1709(84)$ & $1255(62)$ & $454(22)$ & $\chi^{2}($ d.f. $)=81.3(1), P<0.001$ \\
\hline Alcohol $>15$ units per week $(n, \%)$ & $761(38)$ & $555(27)$ & $206(12)$ & $\chi^{2}$ (d.f.) $=0.2(1), P=0.65$ \\
\hline Cannabis use $^{3}(n, \%)$ & $405(20)$ & $299(15)$ & $106(5)$ & $\chi^{2}$ (d.f.) $=2.80(1), P=0.05$ \\
\hline Any childhood life event ( $n, \%)$ & 1499 (74) & $1044(52)$ & $455(22)$ & $\chi^{2}$ (d.f.) $=0.23(1), P=0.88$ \\
\hline Any childhood abuse $(n, \%)$ & $370(18)$ & $256(13)$ & $114(5)$ & $\chi^{2}$ (d.f.) $=0.65(1), P=0.79$ \\
\hline Childhood sexual abuse (CSA) $(n, \%)$ & $176(9)$ & $121(6)$ & $55(3)$ & $\chi^{2}$ (d.f.) $=0.89(1), P=0.76$ \\
\hline Childhood physical abuse (CPA) $(n, \%)$ & $147(7)$ & $98(5)$ & $49(2)$ & $\chi^{2}$ (d.f.) $=0.70$ (1), $P=0.40$ \\
\hline Childhood emotional abuse (CEA) $(n, \%)$ & $45(2)$ & $26(1)$ & $19(1)$ & $\chi^{2}($ d.f. $)=3.1(1), P=0.08$ \\
\hline Death of a loved one $(n, \%)$ & $423(22)$ & $294(15)$ & $129(7)$ & $\chi^{2}$ (d.f.) $=0.14(1), P=0.90$ \\
\hline Victimising events $(n, \%)$ & $448(22)$ & $315(16)$ & $133(6)$ & $\chi^{2}$ (d.f.) $=0.90(1), P=0.76$ \\
\hline School difficulties ( $n, \%)$ & $261(13)$ & $188(9)$ & $73(4)$ & $\chi^{2}$ (d.f.) $=0.74(1), P=0.38$ \\
\hline Any psychosis $(n, \%)$ & $1408(70)$ & & & \\
\hline Any auditory hallucinations ( $n, \%)$ & $469(23)$ & & & \\
\hline Mood congruent & $216(11)$ & & & \\
\hline Abusive/accusatory & $122(6)$ & & & \\
\hline Running commentary & $13(1)$ & & & \\
\hline Visual hallucinations ( $n, \%)$ & $283(14)$ & & & \\
\hline Any delusional beliefs $(n, \%)$ & $1308(65)$ & & & \\
\hline Depressive delusions & $282(15)$ & & & \\
\hline
\end{tabular}

having a history of psychotic symptoms (any childhood event $\chi^{2}=0.02, P=0.46$; childhood abuse $\chi^{2}=0.07, P=0.42$ ) (Table 2).

\section{Delusional beliefs}

There was no significant association between childhood events and having a lifetime ever history of delusions $\left(\chi^{2}=0.19\right.$, $P=0.53$ ). Childhood events were also not significantly associated with persecutory or depressive delusional beliefs. However, subsequent individual analysis showed a significant association between childhood abuse and grandiose delusions, such that individuals with a history of childhood abuse had lower risk of developing grandiose delusions $(\mathrm{OR}=0.68, P=0.03$ ). Analysis of the subtypes of childhood abuse demonstrated that this relationship held true for both childhood sexual abuse
$(\mathrm{OR}=0.66, P=0.02)$ and childhood physical abuse $(\mathrm{OR}=0.66$, $P=0.02$ ). (See Table 2 and 3 for full details.)

\section{Auditory hallucinations}

There was a significant association between childhood events and mood congruent auditory hallucinations $\left(\chi^{2}=8.57, P<0.01\right.$, $\mathrm{OR}=1.55$ ) (Tables 2 and 3 ). This association was driven primarily by the childhood sexual abuse subcategory, which remains significant after controlling for lifetime-ever cannabis use, gender, age at interview and age at onset of illness $(\mathrm{OR}=2.56, P<0.0001)$. Death of a loved one was significantly associated with mood congruent auditory hallucinations $\left(\chi^{2}=6.07, \quad P<0.01\right.$, $\mathrm{OR}=1.37, P<0.05)$, as was experiencing a victimising event $\left(\chi^{2}=9.07, P<0.001, \mathrm{OR}=1.55, P<0.01\right)$.

Table 2 Association between childhood life events and psychosis in bipolar disorder chi-square tests of association (d.f.)

\begin{tabular}{|c|c|c|c|c|c|c|c|c|}
\hline & \multirow{3}{*}{$\begin{array}{c}\text { Any } \\
\text { psychosis }\end{array}$} & \multirow{2}{*}{\multicolumn{3}{|c|}{ Delusions }} & \multicolumn{4}{|c|}{ Hallucinations } \\
\hline & & & & & & Auditory & & \\
\hline & & Persecutory & Grandiose & Depressive & Mood congruent & Abusive/accusatory & Running commentary & Mood congruent \\
\hline Any CLE & $0.02(1)$ & $1.48(1)$ & $2.81(1)$ & $1.16(1)$ & $8.57(1)^{* *}$ & $1.341(1)$ & $1.10(1)$ & $3.02(1)$ \\
\hline Abuse & $0.07(1)$ & $1.62(1)$ & $8.50(1)^{\star *}$ & $0.37(1)$ & $31.87(1)^{\star * *}$ & $16.14(1)^{\star \star * *}$ & $0.20(1)$ & $8.80(1)^{\star}$ \\
\hline CSA & $0.09(1)$ & $1.98(1)$ & $6.28(1)^{* *}$ & $0.26(1)$ & $40.07(1)^{\star \star \star \star}$ & $25.88(1)^{*}$ & $0.73(1)$ & $10.33(1)^{\star *}$ \\
\hline CPA & $0.71(1)$ & $2.43(1)$ & $3.90(1)^{*}$ & $0.01(1)$ & $8.41(1)^{\star *}$ & 3.38 (1) & $0.01(1)$ & $4.89(1)^{*}$ \\
\hline CEA & $3.12(1)$ & $0.87(1)$ & $3.46(1)$ & $1.42(1)$ & $6.38(1)^{* *}$ & $4.31(1)^{\star}$ & $0.29(1)$ & $4.04(1)^{\star}$ \\
\hline Death & $0.01(1)$ & $0.14(1)$ & $0.53(1)$ & $2.47(1)$ & $6.07(1)^{* *}$ & $0.62(1)$ & $0.04(1)$ & $1.77(1)$ \\
\hline Victim & $0.09(1)$ & $0.98(1)$ & $3.46(1)$ & $0.57(1)$ & $9.07(1)^{* * *}$ & $11.26(1)^{\star *}$ & $0.55(1)$ & $1.20(1)$ \\
\hline Family disruption & $0.11(1)$ & $1.99(1)$ & $0.01(1)$ & $0.14(1)$ & $1.4(1)$ & $1.98(1)$ & $0.31(1)$ & $0.40(1)$ \\
\hline School & $0.75(1)$ & $0.17(1)$ & $0.70(1)$ & $0.34(1)$ & $0.20(1)$ & $0.81(1)$ & $0.32(1)$ & $1.71(1)$ \\
\hline
\end{tabular}




\begin{tabular}{|c|c|c|c|c|c|c|c|}
\hline & $\beta$ & S.E. & Wald & d.f. & $\operatorname{Sig} P=$ & Adjusted O.R. ${ }^{a}$ & $95 \%$ C.I. OR \\
\hline \multicolumn{8}{|c|}{ Mood congruent auditory hallucinations } \\
\hline Any CLE & 0.44 & 0.17 & 6.65 & 1 & 0.01 & 1.55 & $1.11-2.16$ \\
\hline Abuse & 0.64 & 0.15 & 17.06 & 1 & 0.001 & 1.90 & $1.40-2.60$ \\
\hline CSA & 0.99 & 0.19 & 24.39 & 1 & 0.000 & 2.56 & $1.76-3.74$ \\
\hline CPA & 0.37 & 0.22 & 2.79 & 1 & 0.09 & 1.45 & $0.93-2.24$ \\
\hline CEA & 0.49 & 0.36 & 1.81 & 1 & 0.17 & 1.63 & $0.79-3.36$ \\
\hline Death of a loved one & 0.31 & 0.15 & 4.18 & 1 & 0.04 & 1.37 & $1.01-1.85$ \\
\hline Victimising events & 0.44 & 0.15 & 8.86 & 1 & 0.003 & 1.55 & $1.16-2.07$ \\
\hline \multicolumn{8}{|c|}{ Abusive and accusatory auditory hallucinations } \\
\hline Childhood abuse & 0.54 & 0.21 & 6.15 & 1 & 0.01 & 1.72 & $1.21-2.63$ \\
\hline CSA & 0.89 & 0.25 & 12.70 & 1 & 0.000 & 2.45 & $1.49-4.00$ \\
\hline CEA & 0.63 & 0.47 & 1.81 & 1 & 0.18 & 1.88 & $0.75-4.72$ \\
\hline Victimising event & 0.07 & 0.23 & 0.91 & 1 & 0.70 & 0.93 & $0.59-1.47$ \\
\hline \multicolumn{8}{|c|}{ Mood congruent visual hallucinations } \\
\hline Childhood abuse & 0.63 & 0.20 & 10.51 & 1 & 0.001 & 1.90 & $1.28-2.80$ \\
\hline CSA & 0.73 & 0.24 & 9.30 & 1 & 0.002 & 2.09 & $1.28-3.35$ \\
\hline CPA & 0.60 & 0.26 & 5.10 & 1 & 0.02 & 1.81 & $1.08-3.04$ \\
\hline CEA & 0.46 & 0.43 & 1.13 & 1 & 0.29 & 1.58 & $0.68-3.70$ \\
\hline \multicolumn{8}{|l|}{ Grandiose delusions } \\
\hline Childhood abuse & -0.39 & 0.13 & 9.07 & 1 & 0.03 & 0.68 & $0.52-0.87$ \\
\hline CSA & -0.41 & 0.18 & 5.44 & 1 & 0.02 & 0.66 & $0.47-0.93$ \\
\hline CPA & -0.42 & 0.19 & 4.88 & 1 & 0.02 & 0.66 & $0.45-0.98$ \\
\hline
\end{tabular}

Childhood abuse was also significantly associated with experiencing abusive/accusatory hallucination content. This association was strongest for childhood sexual abuse $\left(\chi^{2}=25.88\right.$, $P<0.001$, OR $=2.45$ ), however, childhood emotional abuse was also associated with abusive/accusatory hallucinations $\left(\chi^{2}=4.31\right.$, $P<0.05)$, as was experiencing a victimising event $\left(\chi^{2}=11.26\right.$, $P<0.01)$. The association between auditory hallucinations and childhood emotional abuse or victimising events did not remain significant after controlling for lifetime-ever cannabis use, gender, age at interview and age at onset of illness.

\section{Visual hallucinations}

Childhood abuse and sexual, physical and emotional abuse in childhood were significantly associated with mood congruent visual hallucinations $\left(\chi^{2}=8.80,10.33,4.89\right.$ and 4.04 , respectively, $P<0.05-0.01$ ) (Table 2). These associations remained significant after the influence of age, gender and cannabis use is controlled for in childhood abuse $(\mathrm{OR}=1.90)$, sexual abuse $(\mathrm{OR}=2.09)$ and physical abuse $(\mathrm{OR}=1.81)$ (Table 3$)$.

\section{Cumulative effect of multiple childhood events}

The association between experiencing multiple types of childhood life events and psychosis was then considered. The greater the number of different adverse events experienced the greater the odds of developing any auditory, abusive/accusatory or mood congruent hallucinations (Fig. 1).

\section{Discussion}

We hypothesised that, in people with bipolar disorder, adverse childhood events would show a significant association with psychosis, and in particular with symptoms driven by dysregulation of mood (mood congruent delusions and hallucinations) or with persecutory or abusive content. Our hypothesis was only partially upheld. Childhood trauma, or any type of adverse childhood event, was not associated with overall psychosis. We demonstrated significant associations between childhood abuse and hallucinations that are mood congruent or with abusive content. These types of hallucinations remain highly significantly associated with childhood sexual abuse even after controlling for lifetime-ever cannabis use. Significant associations were also found for other types of adverse childhood life events, including death of a loved one, and experiencing a victimising event, including bullying, and hallucinations. We did not find an increased risk of delusional beliefs or persecutory or depressive delusions after any form of childhood event.

The main strength of this study is the detailed clinical information available on a large number of participants. This level of detail, in terms of specific psychotic symptoms and range of childhood experiences, has not been reported before in such a large clinical sample. This has also allowed controlling for the effects of cannabis use, one of the main confounding variables potentially mediating the effects of childhood adversity on the presence of psychosis, gender and age at illness onset. Numerous studies report the frequency of substance misuse following childhood abuse, and the influence of cannabis on precipitating and causing psychosis is now clear. ${ }^{23,24}$ The role of substance misuse in psychosis within bipolar disorder has also been previously proposed as an important factor in mediating poor outcome. ${ }^{25}$ However, over and above this we have found that childhood abuse is a potentially significant factor in the risk for hallucinations, and in addition that experiencing multiple types of childhood event substantially increased risk.

\section{Hallucinations}

When looking at auditory hallucinations specifically, previous research supports the existence of a relationship with childhood trauma, such that in their 2005 review Read et al conclude there is probably a causal relationship between child abuse and hallucinations. ${ }^{2}$ This statement has been criticised as being overambitious ${ }^{10}$ but many papers have reported similar findings. $1,2,6,26$ The majority of this research concerns childhood abuse and the findings tend to show childhood emotional and sexual abuse are 


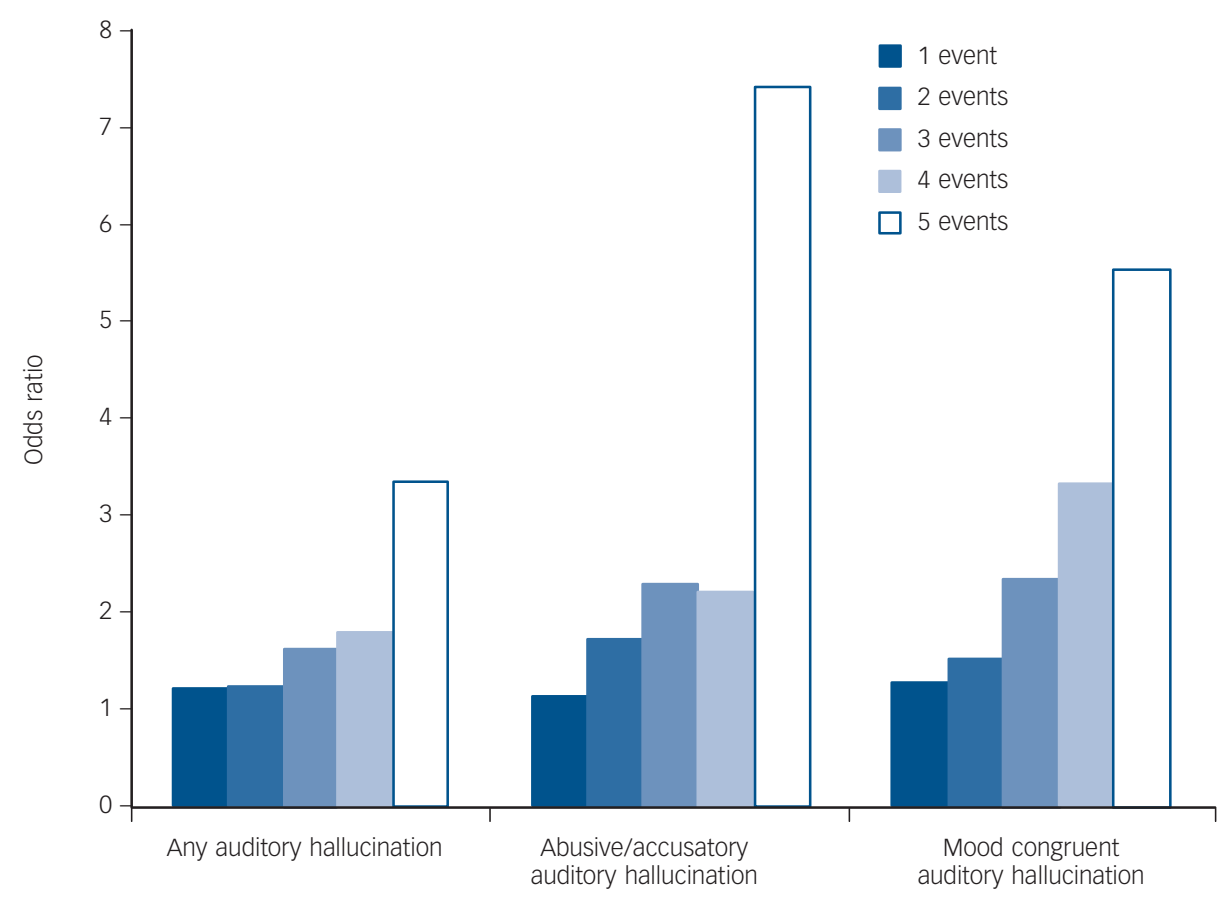

Fig. 1 Auditory hallucinations and number of childhood life event categories

the most notable forms of abuse associated with auditory hallucinations; Daalman et al found that patients with psychosis with auditory verbal hallucinations were three times more likely to be victims of childhood sexual abuse and over five times more likely to have experienced emotional abuse than healthy controls. ${ }^{27}$ Shevlin et al demonstrated that childhood sexual abuse is significantly related to both auditory and visual hallucinations and that experiencing multiple types of trauma increases the likelihood of having hallucinations. ${ }^{28}$

Thus, our results confirm that previous reports are also now relevant to affective psychosis, and add to the weight of evidence of the importance of childhood adversity in increasing the risk of experiencing hallucinations with data from our large clinical sample. Additionally, until now no studies have looked at the form or content of auditory hallucinations in as much detail as our extensive data-set allowed. It is interesting that traditionally Scheiderian first-rank hallucinations of voices speaking in a running commentary were not associated with any type of childhood trauma. It is possible that first-rank symptoms of schizophrenia, linked to a more negative outcome within psychosis, may be less determined by early environmental influences, and possibly have a more genetic, prenatal or epigenetic aetiology. ${ }^{29}$ The strong association between visual hallucinations and child abuse is also interesting. The suggestion may be that this post-traumatic symptomatology has more phenomenological overlap with visual imagery and flashbacks seen within the context of an abusive past. Post-traumatic stress disorder symptoms in psychosis are increasingly recognised, and the phenomenology and pathophysiology may not be as distinct as first proposed. ${ }^{30}$

\section{Delusional beliefs}

The evidence for an association between delusional beliefs and childhood trauma is much less developed than that for auditory hallucinations, since few studies have looked at delusions specifically. Read et al compared 60 patients with psychosis with controls and found the presence of delusions was not related to any form of childhood abuse. ${ }^{31}$ Conversely, Saha et al found that delusional-like experiences were significantly associated with trauma. ${ }^{32}$ Of the small number of papers specifically examining delusions, the majority found no significant association between childhood trauma and delusional beliefs. ${ }^{1}$ Our results, in the largest clinical study of delusions as a discrete symptom, concur with this. We found no significant relationship between any adverse childhood events and depressive or persecutory delusional beliefs. This will thus question the role of childhood trauma in the development of delusions. This is somewhat counterintuitive to the accepted cognitive models of the development and maintenance of persecutory beliefs, which are proposed to be the result of hypervigilance, increased threat to self and cognitive bias of perceived victim status. ${ }^{4,33}$ Many studies investigating the effect of psychological therapies in persecutory ideation, often with mixed results, have this model as their basis. ${ }^{34}$ Our results do not support a role of childhood trauma as a risk for persecutory or depressive delusions in bipolar disorder, suggesting perhaps that the cognitive bias underlying delusions may stem from later experience, or indeed, be more biologically driven.

We did find that childhood abuse had an inverse association with grandiose delusions, a novel finding not previously demonstrated. One possible explanation is that once abuse is experienced, self-esteem or self-belief is so challenged that one is never able to generate grandiose content, even within the context of an elevated mood.

\section{Full range of childhood events and psychosis}

Some previous studies have identified an association between psychosis and wider experiences such as spending time in care, parental loss or separation and victimising events such as bullying. ${ }^{35}$ However, again most studies were community-based and measured subclinical symptoms. Consequently, until now, only childhood abuse has been mainly considered when investigating childhood life events and psychosis. Nevertheless, our data 
support the suggestion that, although childhood abuse, in particular childhood sexual abuse, may be the most significant trauma in relation to later experience of hallucinations, other events such as experiencing a victimising event or the death of a loved one are also extremely relevant.

Bentall et al recently investigated whether specific life events were related to specific psychotic symptoms in a large population-based sample and found that childhood sexual abuse was associated only with hallucinations; alternatively, being brought up in institutional care was associated with 'paranoia'. They proposed the specific associations observed are consistent with current psychological theories about the origins of hallucinations and paranoia. ${ }^{15}$ However, the effect sizes we report suggest that, in those with a diagnosis of bipolar disorder in adulthood, earlier childhood events, and even childhood sexual abuse, may only have a moderate impact on the risk of experiencing hallucinations, and no impact on delusions. A number of other previous studies report significant associations between childhood trauma and psychosis far stronger than this. Most of these studies are from community-based samples with a broad definition of psychosis and classified by self-report. ${ }^{1}$ This may suggest that subclinical or informally diagnosed symptoms, such as hearing brief 'voices', having persecutory-like experiences or 'paranoia', are quantifiably different to formally diagnosed psychotic symptoms, at least within bipolar disorder.

\section{Limitations}

Our findings have limitations that should be taken into consideration. Child abuse was not asked about directly during interview but relied on case-note information and open questions on the CLEQ and BLEQ questionnaires. Therefore the prevalence of childhood abuse in our sample may be underestimated; however, our prevalence rate of near $20 \%$ for childhood abuse is comparable to rates found in other literature. ${ }^{23,25}$ Another important consideration is the retrospective and cross-sectional nature of data collected, which relies on recollection. Many lay people are aware of a link between mental illness and one's upbringing, especially abusive circumstances. Therefore, having been diagnosed with bipolar disorder, participants may look back and scrutinise their childhood years for evidence of abuse, which they otherwise would not have considered. However, the prevalence of abuse reported in our sample does not appear to be in keeping with this. We are careful not to overemphasise the associations demonstrated, and although we can speculate on underlying mechanisms, clearly a direct causal effect has not been demonstrated within this cross-sectional study and further detailed study is required before firm conclusions can be drawn regarding associations between childhood adversity, mood dysregulation and psychosis. Finally, some less common psychotic symptoms, for example disorders of thought or passivity experiences, were not examined.

\section{Implications}

Our findings are consistent with the current evidence that childhood abuse is significantly associated with later auditory and visual hallucinations, but not delusions. This suggests that the relationship between childhood events and psychosis may be symptom-specific. Given the increasing overlap between affective and non-affective psychosis and the increasing importance affect is given in the development of psychosis, this will have relevance through the whole psychosis spectrum. ${ }^{36,37}$ It is clear that childhood adversity has a negative impact on the course of affective disorder ${ }^{38}$ and our results suggest that in part this may be because of a heightened risk of experiencing hallucinations. It is possible that the pathways leading to specific psychotic symptoms differ, with delusions and non-hallucinatory symptoms being influenced less by childhood or early environmental experience. Future research exploring the relationships between childhood experience and psychosis will need to clarify whether this pattern is replicated across the clinical psychosis spectrum. More important, however, may be the need for increasing phenomenological detail of the type and subclass of symptom and the type and the impact of childhood trauma when investigating risk factors for psychosis. We have shown that these risks may well be different for each symptom domain. Increased knowledge here has the potential to help identify those at heightened risk and aid provision of beneficial treatment, for example early and novel treatments for hallucination-related cognitive distortions, such as those targeting inner-speech self-monitoring, in those known to have experienced childhood trauma. ${ }^{39}$ Finally, this detailed knowledge of the specific-symptom risks has the best potential to improve our understanding of what 'causes' psychosis.

\section{Rachel Upthegrove, MRCPsych, PhD, Department of Psychiatry, School of Clinical \& Experimental Medicine, University of Birmingham, Birmingham, Bipolar Disorder Research Network and Early Intervention Service, Birmingham and Solihull Mental Health Foundation Trust, Birmingham; Christine Chard, BMedSc, Lisa Jones, PhD, Department of Psychiatry, School of Clinical \& Experimental Medicine, University of Birmingham, Birmingham and Bipolar Disorder Research Network; Katherine Gordon-Smith, PhD, Department of Psychiatry, School of Clinical \& Experimental Medicine, University of Birmingham, Birmingham, National Centre for Mental Health, MRC Centre for Neuropsychiatric Genetics and Genomics, Cardiff University, Cardiff and Bipolar Disorder Research Network, UK; Liz Forty, PhD, Ian Jones, MRCPsych, PhD, Nick Craddock, FRCPsych, PhD, FMedSci, National Centre for Mental Health, MRC Centre for Neuropsychiatric Genetics and Genomics, Cardiff University, Cardiff and Bipolar Disorder Research Network, UK}

Correspondence: Rachel Upthegrove, Senior Clinical Lecturer, School of Clinical and Experimental Medicine, University of Birmingham, The Barberry, National Centre for Mental Health, 25 Vincent Drive, Birmingham B15 2FG, UK. Email: r.upthegrove@bham.ac.uk

First received 10 Jun 2014, final revision 15 Aug 2014, accepted 10 Sep 2014

\section{References}

1 Varese F, Smeets F, Drukker M, Lieverse R, Lataster T, Viechtbauer W, et al. Childhood adversities increase the risk of psychosis: a meta-analysis of patient-control, prospective- and cross-sectional cohort studies. Schizophr Bull 2012; 38: 661-71.

2 Read J, Os J, Morrison A, Ross CA. Childhood trauma, psychosis and schizophrenia: a literature review with theoretical and clinical implications. Acta Psychiatr Scand 2005; 112: 330-50.

3 van Winkel R, van Nierop M, Myin-Germeys I, van Os J. Childhood trauma as a cause of psychosis: linking genes, psychology, and biology. Can $J$ Psychiatry 2013; 58: 44-51.

4 Garety PA, Kuipers E, Fowler D, Freeman D, Bebbington PE. A cognitive model of the positive symptoms of psychosis. Psychol Med 2001; 31: 189-95.

5 Birchwood M, Gilbert P, Gilbert J, Trower P, Meaden A, Hay J, et al. Interpersonal and role-related schema influence the relationship with the dominant 'voice' in schizophrenia: a comparison of three models. Psychol Med 2004; 34: 1571-80.

6 Janssen I, Krabbendam L, Bak M, Hanssen M, Vollebergh W, De Graaf R, et al. Childhood abuse as a risk factor for psychotic experiences. Acta Psychiatr Scand 2004; 109: 38-45.

7 Aas M, Navari S, Gibbs A, Mondelli V, Fisher HL, Morgan C, et al. Is there a link between childhood trauma, cognition, and amygdala and hippocampus volume in first-episode psychosis? Schizophr Res 2012; 137: 73-9.

8 Marwaha S, Broome MR, Bebbington PE, Kuipers E, Freeman D. Mood instability and psychosis: analyses of British national survey data. Schizophr Bull 2014; 40: 269-77.

9 Bebbington $\mathrm{P}$, Jonas S, Kuipers E, King M, Cooper C, Brugha T, et al. Childhood sexual abuse and psychosis: data from a cross-sectional national psychiatric survey in England. Br J Psychiatry 2011; 199: 29-37.

10 Morgan C, Fisher H. Environment and schizophrenia: environmental factors in schizophrenia: childhood trauma-a critical review. Schizophr Bull 2007; 33: $3-10$. 
11 Galletly C, Van Hooff M, McFarlane A. Psychotic symptoms in young adults exposed to childhood trauma-A 20 year follow-up study. Schizophr Res 2011; 127: 76-82.

12 Kessler RC, McLaughlin KA, Green JG, Gruber MJ, Sampson NA, Zaslavsky AM, et al. Childhood adversities and adult psychopathology in the WHO World Mental Health Surveys. Br J Psychiatry 2010; 197: 378-85.

13 Etain B, Henry C, Bellivier F, Mathieu F, Leboyer M. Beyond genetics: childhood affective trauma in bipolar disorder. Bipolar Disord 2008; 10 867-76.

14 Etain B, Mathieu F, Henry C, Raust A, Roy I, Germain A, et al. Preferential association between childhood emotional abuse and bipolar disorder. J Trauma Stress 2010; 23: 376-83.

15 Bentall RP, Wickham S, Shevlin M, Varese F. Do specific early-life adversities lead to specific symptoms of psychosis? a study from the 2007 The Adult Psychiatric Morbidity Survey. Schizophr Bull 2012; 38: 734-40.

16 American Psychiatric Association. Diagnostic and Statistical Manual of Mental Disorder (4th edn) (DSM-IV). APA, 1994.

17 Parker G, Graham R, Hadzi-Pavlovic D, Mccraw S, Hong M, Friend P. Differentiation of bipolar I and II disorders by examining for differences in severity of manic/hypomanic symptoms and the presence or absence of psychosis during that phase. J Affect Disord 2013; 150: 941-7.

18 World Health Organization. SCAN 2.1: Schedules for Clinical Assessment in Neuropsychiatry. Cambridge University Press, 1999.

19 Brugha TS, Cragg D. The list of threatening experiences: the reliability and validity of a brief life events questionnaire. Acta Psychiatr Scand 1990; 82 77-81.

20 Dube SR, Felitti VJ, Dong M, Chapman DP, Giles WH, Anda RF. Childhood abuse, neglect, and household dysfunction and the risk of illicit drug use: the adverse childhood experiences study. Pediatrics 2003; 111: 564-72.

21 Houston JE, Murphy J, Adamson G, Stringer M, Shevlin M. Childhood sexua abuse, early cannabis use, and psychosis: testing an interaction model based on the National Comorbidity Survey. Schizophr Bull 2008; 34: 580-5.

22 Elklit A, Shevlin M. Female sexual victimization predicts psychosis: a case-control study based on the Danish registry system. Schizophr Bull 2011; 37: 1305-10.

23 Arseneault L, Cannon M, Poulton R, Murray R, Caspi A, Moffitt TE. Cannabis use in adolescence and risk for adult psychosis: Iongitudinal prospective study. BMJ 2002; 325: 1212-3.

24 Arseneault L, Cannon M, Witton J, Murray RM. Causal association between cannabis and psychosis: examination of the evidence. Br J Psychiatry 2004 184: $110-7$

25 Garno JL, Goldberg JF, Ramirez PM, Ritzler BA. Impact of childhood abuse on the clinical course of bipolar disorder. Br J Psychiatry 2005; 186: 121-5.
26 Sheffield JM, Williams LE, Blackford JU, Heckers S. Childhood sexual abuse increases risk of auditory hallucinations in psychotic disorders. Compr Psychiatry 2013; 54: 1098-104.

27 Daalman K, Diederen KMJ, Derks E, van Lutterveld R, Kahn R, Sommer IE. Childhood trauma and auditory verbal hallucinations. Psychol Med 2012; 42 2475.

28 Shevlin M, Dorahy M, Adamson G. Childhood traumas and hallucinations: an analysis of the National Comorbidity Survey. J Psychiatr Res 2007; 41: 222-8.

29 Conus P, Abdel-Baki A, Harrigan S, Lambert M, McGorry PD. Schneiderian first rank symptoms predict poor outcome within first episode manic psychosis J Affect Disord 2004; 81: 259-68.

30 Brunet $\mathrm{K}$, Birchwood $\mathrm{M}$, Upthegrove $\mathrm{R}$, Michail M, Ross $\mathrm{K}$. A prospective study of PTSD following recovery from first-episode psychosis: the threat from persecutors, voices, and patienthood. Br J Clin Psychol 2012: 51: 418-33

31 Read J, Agar K, Argyle N, Aderhold V. Sexual and physical abuse during childhood and adulthood as predictors of hallucinations, delusions and thought disorder. Psychol Psychother 2003; 76: 1-22.

32 Saha S, Varghese D, Slade T, Degenhardt L, Mills K, McGrath J, et al. The association between trauma and delusional-like experiences. Psychiatry Res 2011; 189: 259-64.

33 Udachina A, Thewissen V, Myin-Germeys I, Fitzpatrick S, O'kane A Bentall R. Understanding the relationships between self-esteem, experiential avoidance, and paranoia: structural equation modelling and experience sampling studies. J Nerv Ment Dis 2009; 197: 661-8.

34 Tarrier N. Cognitive behavior therapy for schizophrenia and psychosis: current status and future directions. Clin Schizophr Relat Psychoses 2010; 4 $176-84$.

35 Bebbington PE, Bhugra D, Brugha $T$, Singleton N, Farrell M, Jenkins $R$, et al. Psychosis, victimisation and childhood disadvantage Evidence from the second British National Survey of Psychiatric Morbidity. Br J Psychiatry 2004; 185: $220-6$

36 Birchwood M, Iqbal Z, Upthegrove R. Psychological pathways to depression in schizophrenia: studies in acute psychosis, post psychotic depression and auditory hallucinations. Eur Arch Psychiatry Clin Neurosci 2005; 255: 202-12.

37 Craddock N, Owen MJ. The Kraepelinian dichotomy - going, going . . . but still not gone. Br J Psychiatry 2010; 196: 92-5.

38 Tunnard C, Rane $\mathrm{L}$, Wooderson SC, Markopoulou K, Poon L, Fekadu A, et al. The impact of childhood adversity on suicidality and clinical course in treatment-resistant depression. J Affect Disord 2014; 152-154: 122-30.

39 Moseley P, Fernyhough C, Ellison A. Auditory verbal hallucinations as atypical inner speech monitoring, and the potential of neurostimulation as a treatment option. Neurosci Biobehav Rev 2013; 37: 794-805. 